\title{
Emprendimiento de una microempresa turística (parada turística), en el municipio San Diego, estado Carabobo
}

\section{Entrepreneurship of a Tourist Micro-enterprise (Tourist Stop), in the Municipality of San Diego, Carabobo State}

\Carrillo Yamilett

yamillcarrillo@hotmail.com

ORCID: 0000-0003-4628-7994

Universidad de Carabobo. Venezuela

Artículo recibido en julio 2018 / Arbitrado en agosto 2018 / Publicado en enero 2019

La presente investigación tiene como objetivo proponer un plan de emprendimiento para la creación de una microempresa turística, en el sector turismo (parada turística), en el Remanso Municipio San Diego, estado Carabobo. Metodológicamente es una investigación no experimental, de carácter descriptivo y trabajo de campo; La población está conformada por 2.150 habitantes, con una muestra aleatoria de 45 habitantes de la zona, a la cual se les aplicaron cuestionarios y entrevista para la recolección de la información. A partir de esta se obtuvieron los siguientes resultados: la población residente opina que el turismo es una actividad que debe ser desarrollada en el municipio, pero que hay que mejorarla; en este sentido se propone la creación de la parada turística "El Remanso" como una microempresa comercial y de servicio, que asegure empleo directo e indirecto, orientada en una actividad prioritaria para el país en los actuales momentos.

Palabras clave: Creación, crecimiento desarrollo, emprendimiento, microempresa, turismo, parada turística

The objective of this research is to propose an entrepreneurship plan for the creation of a tourist micro-enterprise, in the tourism sector (tourist stop), in the San Diego Municipality Remanso, Carabobo state. Methodologically it is a non-experimental, descriptive and field work investigation; The population is made up of 2,150 inhabitants, with a random sample of 45 inhabitants of the area, to which questionnaires and interviews were applied to collect the information. From this, the following results were obtained: the resident population believes that tourism is an activity that should be developed in the municipality, but needs to be improved; In this sense, the creation of the "El Remanso" tourist stop is proposed as a commercial and service micro-enterprise that ensures direct and indirect employment, oriented towards a priority activity for the country at the present tim.

Keywords: Creation, increase development, entrepreneurship, micro-enterprise, tourism, tourist stop expenses 


\section{INTRODUCCIÓN}

Hoy en día el emprendimiento viene desarrollándose a través de la historia como factor esencial dentro de la competitividad y liderazgo de los países frente a otros; se ha categorizado como esencia y parte del progreso al servicio de humanidad e innovación de la misma. El emprendimiento es un sector clave y de gran importancia en el desarrollo económico y social de las comunidades donde existen suficientes razones para que el Estado genere políticas y regulaciones que lo incentiven y fortalezcan. Según Draier y Lebendiker (2.013), señalan:

La creación de nuevas empresas son generadas por emprendedores $y$ ayudando a las economías a enfrentar el desempleo, y creación de nuevos puestos trabajo el cual contribuye de un modo positivo y constructivo al bienestar y riqueza de la sociedad, y esto va a traer a través del tiempo una cultura y capacidad en las empresas, una característica del entorno competitivo, variedad, transformación, evolución, innovación, avance científico y del conocimiento. (pág.15).

A nivel mundial, la crisis económica y financiera de la mayoría de los países han provocado que crezca la tasa de mortalidad empresarial hasta llegar a más de la mitad de los negocios creados. Efectivamente, el último informe publicado del GEM (Global Entrepreneur Monitor), que hace referencia a datos de 2.011, afirma que más de la mitad de las empresas (concretamente el 56\%), no supera los 5 años de vida. Es notorio que la administración de las empresas dedican esfuerzo, recursos, dinero para promover y fomentar la creación de empresas y al cabo de 4 ó 5 años más de la mitad de éstas terminan cerrando, esto lleva a reforzar y profesionalizar los servicios de asesoramiento y apoyo de los emprendedores y una mejora del marco legal de estas empresas, así como su fragilidad en las políticas públicas.

Según el estudio realizado por Draier y Lebendiker en el que citan a Gulst y Maritz (2013), donde señalan que:

Los principales problemas en el emprendimiento se pueden agrupar en seis grandes categorías: Estas pueden ser Internas por razones asociadas al propio emprendedor y su ámbito de acción. Entre los factores Internos están las finanzas, gestión, recursos humanos, producto y mercado y externas por factores o variables relacionados con el entorno de la empresa. Entre los factores externos: finanzas, gobierno $y$ entorno, producto y mercado. Se evidencia que las principales causas del problema están en el interior de la organización y, por ende, se refieren a aspectos en los que el emprendedor puede incidir. Al final las habilidades y la experiencia en gestión son mucho más relevantes que el entorno y su evolución. (Pag.25)

Es por ello que, los problemas financieros y deuda excesiva, son a causa del uso inapropiado del dinero, poco acceso a capital, cambios en la economía local y el elevado coste de financiamiento. Al igual que, los problemas de gestión son debido a estrategia de administración pobre, cualidades y habilidades de gestión inadecuadas, equipo de administración, plan de negocios pobre o inexistente, bajo control de costes, precios y distribución, problemas de liderazgo, personas claves realizan trabajos más allá de lo que corresponde.

En cuanto a los problemas de producto y mercado; éstos surgen en torno a las necesidades de mercado no focalizadas, relaciones pobres con proveedores $\mathrm{y} / \mathrm{o}$ vendedores, tamaño de mercado, mercado externo pobre sustitutos, alternativas, 
competidores indirectos, desconocimiento de los clientes, falta de experiencia, bajo crecimiento del mercado, imposibilidad de acceso o renuncia a mercados mayores, mercado limitado o deteriorado, barreras de entrada. Con relación a los problemas de recursos humanos que se generan por la mala elección y equivocada de partners, reclutamiento de personas incompetentes, problemas personales (muerte, divorcio, etc.); en donde existe la necesidad de mejorar y de llevar a cabo las propuestas.

Por lo tanto, los problemas asociados al papel del Gobierno y del Entorno son a consecuencia de condiciones del entorno desfavorables, regulaciones y disponibilidad de fondos del Gobierno y problemas legales. De tal manera, la situación actual de Venezuela, descrita con las evaluaciones efectuadas por diversos organismos internacionales de reconocida probidad y capacidad técnicas. Según el índice Global de Libertad Económica 2.011, elaborado por el Fraser Institute en colaboración con Cedice Libertad, Venezuela ocupó el puesto 139. (El Universal. 21/09/11).

Según el Heritage Foundation, un instituto de investigación y educación integrado por un grupo de expertos, patrocinados por el Wall Street Journal; cuya misión consiste en formular y promover políticas públicas basadas en los principios de la libre empresa, gobierno limitado, y la libertad individual. Clasifica en una escala 0 a 100 las economías de 179 países de acuerdo a la libertad económica, en base a subíndices de libertad económica, seguridad y prosperidad, según investigaciones de organismos internacionales calificados y la información de los propios países.

Los diez primeros países del ranking 2.011 son: Hong Kong 89,7, Singapur 87,2, Australia 82,5, Nueva Zelandia 82,3, Suiza 81,9, Canadá 80,8, Irlanda 78,7, Dinamarca 78,6, Estados unidos 77,8, Bahréin 77,7. Los diez últimos países del ranking 2.011 son: Timor Oriental 42,8, Irán 42,1, Congo, República Democrática del 40,7, Libia 38,6, Birmania 37,8, Venezuela 37,6, Eritrea 36,7, Cuba 27,7, Zimbabue 22,1, Corea del Norte 1,0 .

En lo anteriormente expuesto se observa que, la posición de Venezuela con respecto a otros países está en desventaja en cuanto a sus políticas públicas basadas en los principios de la libre empresa, gobierno limitado, libertad individual, seguridad y prosperidad. Venezuela ocupa una posición en economía en 175 de 179 países en estudio y se clasifica en una escala de 0 a 100 en $37,6$.

El informe de Competitividad Global 2.013 2.014, señala el estudio de la capacidad de competir internacionalmente de cada país, mide, las instituciones, la infraestructura, el clima empresarial, la educación, la preparación tecnológica y la innovación de cada uno de los países. El país latinoamericano que ocupa el puesto más alto en el ranking es Chile (puesto 34), Panamá (40), Barbados (47), Costa Rica (54), México (55), Brasil (56), Perú (61), Colombia (69), Ecuador (71), Uruguay (85), Guatemala (86), El Salvador (97), Bolivia (98), Nicaragua (99), y Argentina (104). Al final del ranking, entre los países menos capacitados para competir en el mundo, figuran Venezuela (134) y Haití (143).

De acuerdo al análisis del informe de Competitividad que mide la capacidad de competir internacionalmente cada país, el cual mide las instituciones, la infraestructura, el clima empresarial, la educación, la preparación tecnológica y la innovación de cada uno de los países, Venezuela se encuentra entre los países menos capacitados para competir en el mundo, estando en posición (134). Según Farías (2.014), señala que: 
La centralización del poder político y económico ilimitado en el Estado, a medida que crece la población, los problemas y la complejidad de la administración de los asuntos públicos, convierte al gobierno, en un generador de pobreza, opresión, atraso y una administración deficiente, donde no pueden gerenciar con eficiencia las innumerables necesidades y complejidades del desarrollo del país.(pág. 57)

Cabe destacar que los problemas que presenta Venezuela a nivel nacional en lo político, económico y social, a través de su administración, ha traído como consecuencia la pobreza, el desempleo, pérdidas de empresas, inseguridad, donde se evidencia la pérdida de calidad de vida de las personas en general, pérdida del poder adquisitivo y material, dificultad para hacer realidad una iniciativa empresarial o emprendimiento, donde la microempresa debido a las pocas políticas públicas, el financiamiento es menor y el desarrollo y el crecimiento de las mismas es poco probable.

Aunque Venezuela es un país en dependencia de una economía petrolera, existe otra fuente de economía, el turismo siendo este un fenómeno contemporáneo que se ha desarrollado a lo largo de la historia aparejado con los medios de comunicación. Hay muchas causas que hacen que el turismo haya tomado mundialmente importancia. En los países Europeos y en Estados Unidos el turismo, como movimiento más o menos interesante de volumen de personas, toma fuerza al establecerse el ferrocarril como medio de transporte.

El Turismo es un factor de primer orden como vía alterna de desarrollo económico. Tal como lo señala Correa (2013), define el turismo como:

Un fenómeno de naturaleza compleja y multidisciplinaria, tal como lo hemos afirmado con anterioridad, el turismo que proponemos sólo puede ser abordado como actividad transdisciplinaria, con diversas dimensiones a considerar: cultura, económica, social, ecológica, educativa y ética, entre otras que pudieran considerarse para el establecimiento de las necesarias conectividades del turismo con las demás ramas del saber humano. (pág. 50)

En lo anteriormente expuesto, se considera el turismo como un fenómeno de naturaleza compleja y multidisciplinaria como la cultura, economía, social, ecológica, educativa y ética, entre las cuales la cultura implica crear la relación directa del turista con las comunidades, cultura y conocimiento conjuntamente con su medio ambiente.

A pesar de que el turismo viene desarrollándose a través de la historia en forma paulatina, también presenta sus problemas y debilidades, según Correa (2013:45) "la planificación turística en América Latina se comenzó con desarrollos de tipo urbano, definidos por criterios de planificación físicoespacial".

La Organización de Estados Americanos, mediante su Programa de Desarrollo turísticoSistema CICATUR, se formuló los primeros planes de desarrollo integral del Turismo en Latinoamérica. Fueron elaborados bajo la concepción teórica de la planificación normativa, intentando integrar al turismo como una actividad productiva en el desarrollo de la región. No obstante, a pesar de estos esfuerzos, los requisitos de las entidades financieras 
internacionales aumentaron el interés en la planificación física en América, dando origen a grandes proyectos de desarrollo turísticos, muchos de los cuales no fueron ejecutados.

Es evidente porque estos proyectos y su planificación se hicieron desvinculados de las comunidades receptoras. Sólo operaban organismos como la O.E.A., Banco Mundial, en conjunción con grandes inversionistas internacionales. Por lo demás, los proyectos turísticos planteados no estaban articulados a los planes de desarrollo de los países, lo cual no favoreció el éxito de dichos proyectos.

Cabe destacar, que en América Latina hay países con bondades turísticas y que apoyan sus economías en este sector; Venezuela es uno de ellos, aunque es un país dependiente de la actividad petrolera, sin embargo, aprovechando los cambios coyunturales de la nueva estrategia económica del gobierno venezolano, cuando se presenta una economía como la que hoy se tiene, donde la mayoría de los sectores se ven afectados, otros sectores están empezando a presentar ventajas comparativas, siendo el turismo una de las actividades donde el emprendimiento puede ser una de las raíces de ese gran árbol productivo que crecerá en la nación; y lo más probable es que madurándose una conciencia micro empresarial, se logre comprender que también la colectivización puede ser productiva, aunque parezca una paradoja.

El año 2.013, según el Ministerio del Poder Popular para el Turismo, el ingreso turístico interno en el período 2.005-2.013, se ubicó en un 96,12\%, reflejando un aumento en ascenso cada año en dicha actividad. El turismo en Venezuela se ha caracterizado por una tendencia predominante del turismo emisivo sobre el turismo receptivo. Se evidencia que en los últimos dieciséis años (periodo: 1.997-2.013), se ha mantenido un 3,95\% de participación en la economía del país. En consecuencia, la balanza del gasto turístico para Venezuela es deficitaria.

En el Plan Estratégico Nacional de Turismo (2.009-2.014), se muestran las debilidades en materia de turismo, en evidencia de una falta de planificación de los destinos turísticos y de políticas públicas junto a la carencia de los servicios básicos de infraestructura turística, inadecuada plataforma tecnológica y fallas en el manejo de información turística, lo que trae como consecuencia una baja en la balanza del turismo internacional.

En los planes de los últimos periodos de gobierno, el emprendimiento y el turismo se han venido fortaleciendo. La creación de Organismo Financieros (FONDEMI) y otros fondos; la puesta en vigencia de la legislación necesaria casi siempre vía decretos presidenciales por habilitación; el apoyo de la banca pública y privada, con intereses preferenciales y cuotas obligadas para con microempresas (emprendimiento) y el Turismo, así como a cooperativas, consejo comunales, comunas y otras forma asociativas, han sido manifestaciones determinantes de apoyo al emprendimiento.

La fórmula emprendimiento turismo se perfila como forma de desarrollar proyectos socio-productivos en todas sus modalidades: Información, alimentos, cultura, historia, religión, protocolo, naturaleza, aventura, transporte, agroturismo y ecoturismo, entre otros.

Actualmente en el Estado Carabobo, el sector turismo, tiene una importancia en la economía de los distintos organismos a nivel estadal y municipal, pero aun existiendo políticas públicas dirigidas al sector turismo los agentes turísticos y especialistas en la materia no han tenido la disposición y por ende la cultura en materia de turismo. Realmente la falta de motivación y estrategias efectivas que generen 
riquezas materiales y humanas a las comunidades se ha dejado de percibir un ingreso que permita el desarrollo social y económico de la región.

Estos problemas políticos, económicos y sociales pasan del nivel nacional, al nivel regional y a su vez a nivel municipal. San Diego es uno de los municipios del estado Carabobo; la problemática del sector turismo en este municipio se evidencia por falta de asistencia técnica en las políticas públicas existentes y políticas de financiamiento, a pesar del gran potencial de recursos con que cuenta este municipio no se desarrolla esta fuente de riqueza que es una de las vías para el desarrollo de las comunidades, y pueblos cercanos a la misma.

En materia de turismo, las comunidades deberían hacer turismo comunitario, estudiar e implementar la cultura de turismo, dedicar a tomar esta actividad como otra fuente de ingresos para el mejoramiento de la calidad de vida de los mismos. Es importante destacar que los pueblos deben resaltar los valores y cultura del turismo para complementar conjuntamente con sus idiosincrasias su legado. Emprender, hacer turismo aunque tenga un grado de incertidumbre y de riesgo tomando en cuenta el apoyo de los entes de desarrollo regional y local.

En relación al gran potencial que se evidencia en este municipio es oportuno buscar la manera idónea de explotar su atractivo turismo de manera que a través del mismo se puedan generar recurso que permitan el desarrollo y evolución del municipio. De esta manera, surge la iniciativa de proponer un emprendimiento para la creación de una microempresa turística, (parada turística), en el municipio San Diego, estado Carabobo.

\section{MATERIALES Y METODO}

Para el desarrollo de la presente investigación, metodológicamente se llevó a cabo a través de un diseño no experimental, ya que es donde el investigador observa la realidad de los fenómenos tal como son para después analizarlos. En cuanto al tipo de investigación se desarrolló bajo la modalidad del estudio de campo, ya que permite evidenciar los datos necesarios en la recolección de información donde se desarrolla el problema y directamente de la realidad. En este caso, al presentar una propuesta de emprendimiento de creación de microempresa turística (Parada Turística), en el municipio San Diego, Estado Carabobo, se hará el trabajo de campo seleccionado con los métodos y procedimientos respectivos para poder dar solución y respuesta a los objetivos de la investigación.

La población, está conformada por los 2.156 habitantes del Remanso, san diego Estado Carabobo, tomando en cuenta que una población es el conjunto de los elementos que poseen características comunes, las cuales se estudian y dan origen a los datos de la investigación. En cuanto a la muestra la conformaron 45 personas tomadas de manera aleatoria, considerando que la muestra es una porción o un porcentaje de la población propiamente dicha, de la cual se va a tomar $u$ obtener la información de la realidad y donde el investigador se basa para tomar decisiones que afectan al proyecto. En relación a la técnica y recolección de datos, en el presente estudio se empleó la encuesta y entrevista. Aplicando un cuestionario como instrumento el cual estuvo conformado por 6 preguntas cerradas.

\section{RESULTADO Y DISCUSIÓN}

Una vez aplicado los instrumentos estos dieron como resultado que el municipio San Diego del estado Carabobo, tiene un gran potencial turístico, sin embargo este aspecto no es prioridad en las políticas públicas en ninguno de los niveles del gobierno. En 
consecuencia se observa que aunque existan políticas públicas a nivel municipal; éstas no se aplican, generando debilidades y dificultades en el turismo.

Los habitantes señalan que no existe una planificación para el desarrollo turístico ya que no se cuenta con mecanismos de inversión que ayuden a generar y promover el turismo en la zona. Sin embargo a pesar de que no se cuenta con un plan de desarrollo turístico, existen infraestructura turística pública y privada creadas hace tiempo atrás para el buen desarrollo del turismo, tales como como parques, plazas, iglesias, cuentan con un mantenimiento óptimo de las instalaciones de cada uno de los sitios turísticos en San Diego, estado Carabobo.

Así mismo señalaron que los emprendimientos en San Diego son promovidos por el sector privado y gracias a ello existen sitios turísticos en el que la población puede desarrollarse y generar sus propias riquezas. Los resultados demuestran, que es necesario establecer políticas de financiamiento para el sector turismo ya que generaría más crecimiento en la población y a su vez incentivaría a los futuros microempresarios en emprender en este sector del turismo.

De acuerdo a todo lo señalado anteriormente se plantea técnicas que permitan desarrollar el emprendimiento de una microempresa turística en el municipio San Diego, estado Carabobo Venezuela, a través de una parada turística; la cual permitirá fortalecer la promoción turística a nivel local, promover los sitios turísticos, desarrollo como actividad turística, promover el crecimiento del turismo social y comunitario. Así como también promover y garantizar su rentabilidad en el marco del motor turismo, donde la meta es lograr una economía productiva diferente a la economía de la actividad petrolera. Es una alternativa de asegurar el trabajo para el profesional decidido a incursionar en un emprendimiento, orientado en una actividad prioritaria en los actuales momentos.

Esta parada turística, una vez que sea concluida y debidamente dotada, ofrecerá los servicios de información turística, gastronomía municipal y regional; venta de exquisiteces de las diferentes regiones más sobresalientes en turismo en Venezuela, venta de artesanía de las diferentes zonas turísticas, ya sean del municipio, como del estado Carabobo y de otras regiones del país; venta de periódicos y revistas, y en conexión con otros servidores de turismo, ofrecer paquetes turísticos, giras, visitas, boletos de viajes, entre otras.

Se puede señalar, que este proyecto micro empresarial es original por su innovación y que podrá ser modelo en emprendimiento para la actividad turística. No es notorio este tipo de paradas en el municipio hasta el punto de que en el futuro se constituya una Red Nacional de Paradas Turísticas; este servicio de esta forma, será soporte para un crecimiento sostenido del turismo y la posibilidad de empleo para personas residentes en el municipio y de otras partes del estado Carabobo.

Los servicios serán ofrecidos por personas debidamente entrenadas, identificadas y bien presentadas con trato cordial y diligente. Se puede señalar que este proyecto micro empresarial es original por su innovación y que puede ser modelo en emprendimiento para la actividad turística y que en el futuro se constituya una Red Nacional de Paradas Turísticas; de esta forma será soporte para un crecimiento sostenido del turismo y la posibilidad de empleo para personas residentes en el municipio.

\section{CONCLUSIONES}

Luego de presentar los resultados de las investigaciones, y ser debidamente analizados, 
se presentan las conclusiones, en relación al diagnóstico en el sector turismo en San Diego, se concluyó que el turismo en el municipio San Diego no está lo suficientemente desarrollado debido a que un gran porcentaje de personas hacen turismo fuera del municipio, lo que genera también la carencia de puesta en marcha de las políticas públicas existentes en el municipio. Por consiguiente, los problemas o fallas en este sector no han sido prioridad de ninguno de los niveles de gobierno.

Por otra parte, al emprendimiento para el desarrollo de microempresas turísticas, se concluyó tomando en cuenta el modelo del GEM, para que se desarrolle una buena actividad emprendedora se deben conjugar los siguientes elementos:

1. Las condiciones generales del país: gobierno, infraestructura, mercado financiero, instituciones.

2. Las condiciones específicas para el desarrollo de los emprendedores, como por ejemplo: acceso al capital, apertura del mercado interno, acceso a la infraestructura, programas de gobierno, educación y entrenamiento, transferencia de tecnología, normas culturales y sociales, infraestructura legal y comercial.

3. El crecimiento económico nacional (hay que considerar que en líneas generales el número de nuevos emprendimientos crece cuando lo hace la economía de un país).

4. La dinámica de los negocios (cantidad de empresas que nacen y se expanden, cantidad de empresas que se achican y mueren).

5. Las oportunidades de negocios (existencia objetiva y percepción).

6. Las capacidades emprendedoras (actitud emprendedora, aptitud emprendedora y ambición emprendedora).
En cuanto al diseño de la microempresa turística (Parada Turística), en el municipio San Diego, estado Carabobo, se pondría en marcha y en funcionamiento dicha parada para el servicio turístico con el fin de que contribuya con el desarrollo económico y social por lo que se debe adecuar la parada de transporte colectivo para convertirla en una parada turística, luego dotar la parada turística de suministros requeridos para brindar un buen servicio a los usuarios y usuarias del municipio y finalmente iniciar el funcionamiento de la parada turística para lograr atender al usuario de información requerida en el municipio San Diego, estado Carabobo.

\section{REFERENCIAS}

Arias (2006). El Proyecto de Investigación. Introducción a la Metodología Científica (5ta ed.). Editorial Espíteme. Caracas

Balestrini (2006). Como se Elabora el Proyecto de Investigación (7ma ed.). Editorial BL Consultores Asociados. Caracas

Barnuevo (2.013). Emprendimiento de un Plan de Negocio Turístico de Hacienda Yangana Lodge de la Parroquia Yangana. Cantón Loja: Universidad Nacional de Loja en Ecuador

Correa (2013). Turismo Sostenible. Un Debate para la Conservación Multigeneracional de Nuestros Recursos (lra ed.). Editorial Corporación ASM, C.A. Valencia, Venezuela

Cruz y Valls (2.012). Causas de Fracaso de los Emprendedores. Editorial Gesbiblo, S. L. Primera Edición. España

Draier y Lebendiker (2.013). Claves para Emprendedores: Conceptos Básicos para Planificar y Desarrollar tu Proyecto. Primera Edición. Buenos Aires.

Farías (2.014) Crisis Venezolana. Causas y Soluciones Estructurales. Primera Edición. Venezuela 
Flames (2012). Trabajo de Grado Cuantitativo y Cualitativo (2d Ed.). Editorial de la universidad Bolivariana de Venezuela. Caracas

Hurtado y Toro (2005). Paradigmas y Métodos de Investigación en Tiempos de Cambio. Editorial SEC, S.A

Jurado, Leal y Oberto (2.014). Estrategias de Emprendimiento para la Creación de Microempresas en el Estado Carabobo no Dependientes de Importaciones para los Estudiantes de 9no. Semestre de la Facultad de Ciencias Económicas y Sociales de la Universidad de Carabobo. Venezuela

Ley de Fomento del Turismo Sustentable como Actividad Comunitaria y Social. Decreto № 1.442. Gaceta Oficial Extraordinaria de la República Bolivariana de Venezuela № 6.153, 18 de noviembre de 2014. Caracas

Ley de Inversiones Turísticas y del Crédito para el Sector Turismo. Decreto № 1.443. Gaceta Oficial Extraordinaria de la República Bolivariana de Venezuela № 6.153, 18 de noviembre de 2014. Caracas

Ley Orgánica de Ciencia, Tecnología e Innovación (L.O.C.T.I.). (2005)
Ley Orgánica del Turismo. Decreto $\mathrm{N}^{\circ} 1441$. Gaceta Oficial Extraordinaria de la República Bolivariana de Venezuela № 6.152, 18 de noviembre de 2014. Caracas.

Londoño (1.998). Gerencia y Recursos Humanos. Editorial Norma S.A. Bogotá. Colombia

Marcano (2.014). Políticas de Financiamiento Dirigidas a Incentivar el Emprendimiento en el Sector Turismo de las Comunidades de Naguanagua, Estado Carabobo. Venezuela

Palella y Martins (2.012). Metodología de la Investigación Cuantitativa. Editorial Pedagógica de Venezuela (lr. ed.). Caracas

Silva (2.008). Metodología de la Investigación (Elementos Básicos). Editorial CO-BO. Caracas

Veciana (2005). La Creación de Empresas. Un Enfoque. Ed. Electrónica disponible en internet: www.estudios.lacaixa.es

Williams y Ortega (2.009). El Informe de Investigación: Estructura y Estilos Bibliográficos. Editorial Grafiexpress Digital. (lera. Ed.). Caracas 